\title{
Through the Aleph: A Glimpse of the World in Real Time
}

\author{
Jing Zhou \\ Monmouth University \\ New Jersey, USA \\ jz67867009@yahoo.com
}

\section{INTRODUCTION}

Through the Aleph is a net art project offering an unprecedented visual and interactive experience where many places on Earth and in space can be seen simultaneously in an instant. It visualises the diversity of human civilisations (microcosm) and the unity of humanity without borders in the everchanging universe (macrocosm); it draws the connections between individuals and the global environment, Earth and outer space, eternity and time, and art and science. With an unexpected approach to surveillance cameras and global networks this meditative web project uses live data to create an abstract landscape in an open source environment. It not only embraces the dream of peace on Earth but also explores the bond between humankind and nature through time and space in the present moment.

* The project title was inspired by two great literary works - The Aleph and Through the Looking-Glass. Perhaps the computer screen is our modern day looking-glass, and we are all Alice as we peer through our screens at an alternate reality.

\section{STATEMENT}

What is an Aleph? In his short story The Aleph (1945), Argentine author Jorge Luis Borges described that an Aleph is one of the points in space that contains all other points - the single gigantic instant where millions of acts in the unimaginable universe can be seen simultaneously from every point and angle (Borges 1970).

Although a real Aleph might never be found, following Borges's vision Through the Aleph is a net art project offering an unprecedented visual and interactive experience where many points on Earth and in space can be seen simultaneously in an instant. It contains 142 live surveillance webcams selected from seven continents with multiple cultures, real-time water temperatures of four oceans - Arctic, Atlantic, Indian, and Pacific Oceans - which play a crucial role in shaping the global climate change, 12 constellation maps alternating dynamically every month, a thin rotating line in the background representing the passage of time - as the line moves like a second hand as it sweeps around the face of a clock, the latest NASA solar image updated every 12 hours, NASA International Space Station videos of Earth, and satellite time-lapse imagery of the solar system planets. (Figure 1-2) The video component of this project consists of 12 days of time-lapse screenshots of the artwork in 12 months (one day per month during 2016-2017) that captures not only the shift of day and night, but also the change of seasons - the infinite and transitory nature of life on Earth.

Inspired by the life changing power of seeing Earth from space, Through the Aleph visualises a pair of opposites through the global reach of technology the diversity of human civilisations (microcosm) and the unity of humanity without borders in the everchanging universe (macrocosm). Although in recent times some creative and research works have used IP camera live stream and their linkage to the social environment also with the political scope to highlight the implications of this technological Panopticon, the artwork here presented utilises surveillance cameras and global networks from a grand viewpoint to observe people, environment, and space within a philosophical and literary framework. Using live data to portray the Earth's pulse and human existence, this meditative web project creates an abstract landscape in an open source environment, reveals an emerging totality visible to the human eye through distant points of perceptions, and gathers all realities into the glimpse of the Aleph, where we could experience humanity as one in the unimaginable space therefore, the unity in infinity. 


\section{REFERENCES}

Borges, J. (1970) The Aleph and Other Stories.

New York: E. P. Dutton \& Co., Inc.

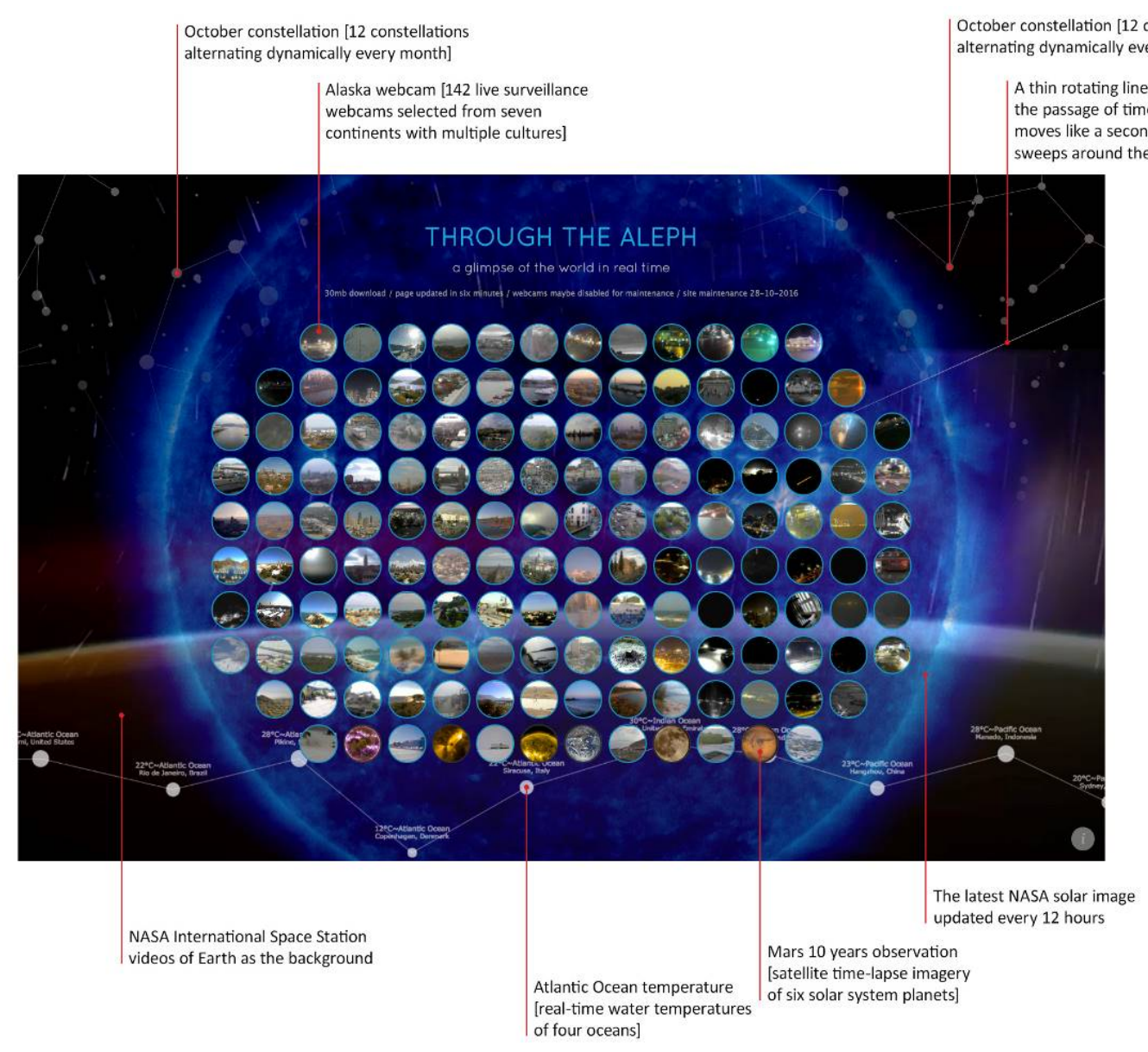

Figure 1: The Diagram of "Through the Aleph"

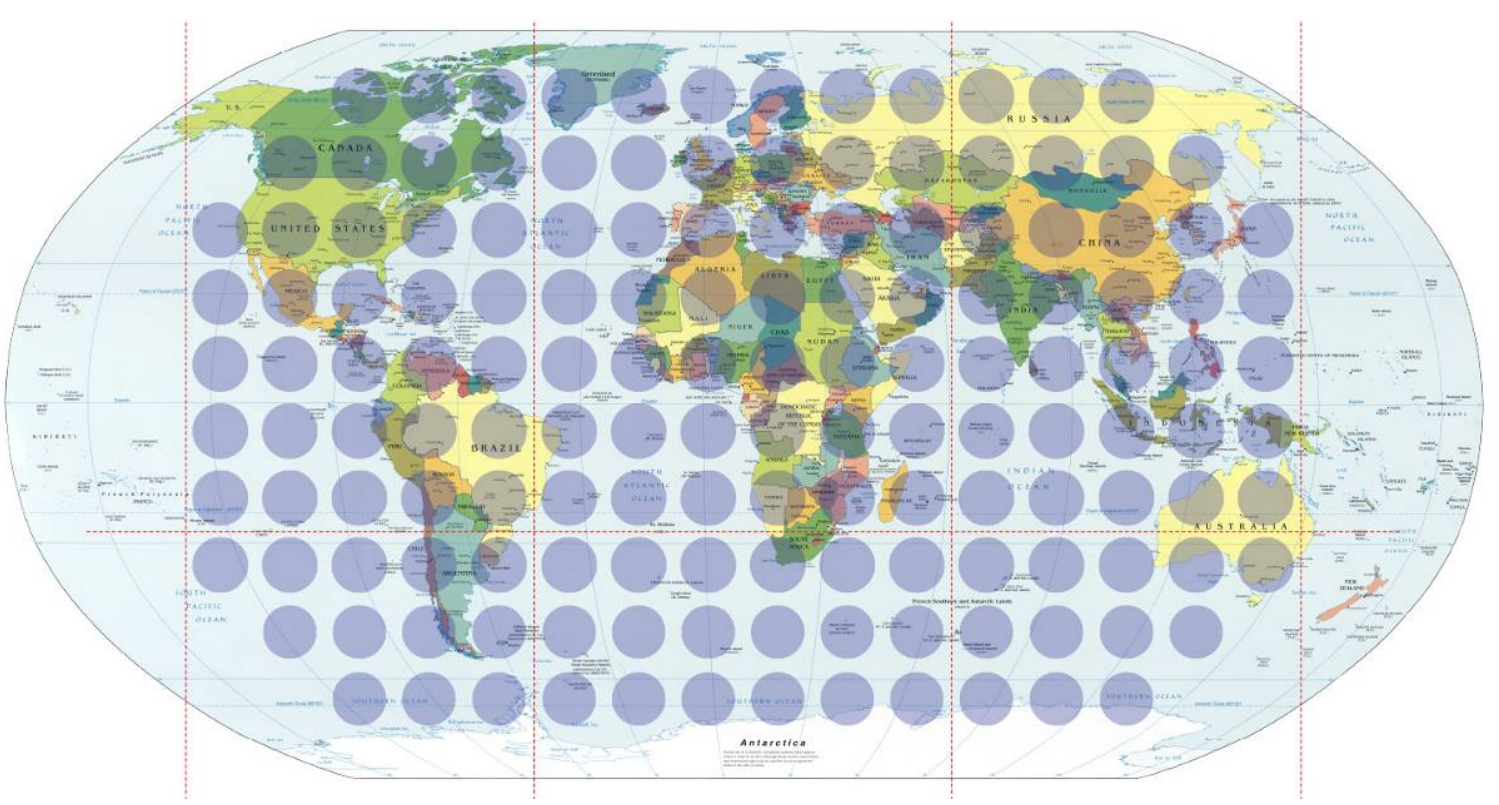

Figure 2: Webcams' Geographic Distribution 